\title{
Influence of Router Buffer Size on TCP Execution
}

\author{
D. Arjun Mitra Reddy \\ Department of Computer Science, University of Central Florida, Orlando, Florida, USA \\ Email: arjunmitrareddy@gmail.com
}

\begin{abstract}
In this paper, Internet Protocol router buffer size's impact on the TCP execution is dissected. TCP StartUp, TCP Flows and High Speed TCP (HSTCP) Performance-these are the different variables considered in breaking down the execution. In this paper, a general investigation of the Slow Start (SS) Phase is introduced as a capacity of the system and TCP parameters, and after that a correlation of the execution on ways with expansive and little buffers is given. Next the buffers' sizing task will be considered for TCP Flows. Finally, the impact of buffer size on the throughput of High-speed TCP is concentrated on.
\end{abstract}

Index Terms-Blockage Control, High Speed Networks, Transmission Control Protocol, Throughput Analysis, Buffer Sizing, Real-Time Traffic Loss

\section{INTRODUCTION}

The internet's transport protocol is TCP [9, 12]. Utilizing a blockage window (W), it controls the stream of packets as a capacity of network blockage. W speaks to the most extreme number of packets the source can transmit without the receipt of any affirmation (ACK) from the object. Toward the start of a TCP association, a Slow Start stage (SS) is called to build W rapidly and easily. It is trailed by a slower build stage called Congestion/Blockage Avoidance (BA) [9]. The changing from SS to CA happens at a window called the SS limit (Wth) which is the source appraisal of the network system limit. By channel size or by network system limit it is implied that in the continuation the most extreme number of packets can be fit on the way between the source and the objective.

The primary SS stage is known to damage the execution of short TCP exchanges particularly on long defer connections, for example, connections in the satellites $[1,2,3]$ and this issue is one amongst the primary issues talked about in the TCPSAT working gathering of the Internet Engineering Task Force. The principal reason is that on long defer joins, $\mathrm{W}$ needs quite a while to achieve huge qualities. Amid this time, network system assets are underutilized. The arrangement proposed to this issue comprises in quickening the window increment amid SS. By Byte Counting [1] it has been known that the quantity of parcels secured by an ACK while expanding the window is considered as opposed to the quantity of ACKs. The point is to beat any postponement of ACK component at the end of the line. It is likewise observed that the Large Initial Window recommendation [2] comprises in beginning the association with a window bigger than 1 parcel, but less than 4 full parcels.
The next issue is related to the losses in the first SS stage. On the off chance that Wth is not situated properly toward the start of this stage, then before changing to CA itself the blockage can occur. This blockage brings about numerous from the same window as a result of the quick window increment amid SS. A long Timeout is obliged to recoup from these misfortunes [6]. This Timeout is trailed by different diminishments in $\mathrm{W}^{\text {th }}$ and another SS stage. A strategic distance from the effect of these undesirable losses on the execution needs to be maintained. For this, a recommendation has been made in [8] to gauge a more precise quality for the SS edge toward the start of the association. Ordinarily, this edge is situated to the window publicized by the collector. The creator in [8] proposes to utilize the stream of Acks toward the start of SS to gauge the Bandwidth-Delay Product (BDP) of the way and after that to set Wth to this quality.

In this paper the assignment of estimating buffers for TCP streams in 802.11e WLANs is likewise considered. Center is accentuated on the commonplace arrangement situation where a framework mode WLAN is arranged with the Access Point (AP) going about as a remote switch between the WLAN and the Internet. TCP activity is of specific significance in such Wlans as it presently conveys the incredibly larger part (more than 90\% [17]) of system movement. Impacts of buffer related issues in Wlans have received little consideration in the writing. Exemptions incorporated[14] which demonstrate that fitting buffer estimating can restore TCP transfer/download injustice, and [16] in which TCP execution with settled AP support sizes and $802.11 \mathrm{e}$ is examined. The current paper amplifies the past chip away at buffer measuring for voice traffic [13], and is the first work concentrating on how to tune cradle sizes for TCP activity in 802.11e WLANs.

Next we consider the instance of HSTCP. In high bandwidth delay item networks, because of improbable demands of TCP's reaction capacity, TCP cannot open its window sufficiently expansive to use the accessible data transmission. TCP is upgraded to High Speed TCP [19], to address the above mentioned central issue, which adjusts the window progress for high blockage window (low loss) administration. After a certain window limit, High Speed TCP expands its blockage window forcefully to get the accessible data transmission and on misfortunes, it decreases the window gradually to stay smooth. Since High Speed TCP's window progress go live just everywhere window (low loss) administration, High Speed TCP does not change TCP conduct in situations with high to gentle blockage (common for Low Speed systems.) In high bandwidth deferral systems in which High Speed TCP 
sends blasts of substantial number of packets, the measure of buffer accessible in the bottleneck switch is a paramount issue to keep the switch profoundly used amid blockage periods. The current designs oblige router producers to give a buffer whose size is tantamount to the bandwidth delay product (BDP) of the way which scales straightly with line speeds. As the connection limit increments to many Gbps, giving such a colossal buffer might definitely build the expense of the routers and force mechanical issues, for example, dispersion of heat, on-chip board space and expanded memory access inertness. An expansive buffer expands delay and delay fluctuation which antagonistically influences constant applications (e.g., feature diversions, gadget control and feature over IP applications.) So in this manner it is very critical to find the impacts of buffering on High Speed TCP execution, for example, throughput, merging to decency and communication in the vicinity of RED. Prior suggestions have been surrounding around enhancing the execution of High Speed TCP or different variations to scale TCP in High Speed situations, e.g., [22], [23], [24]. None of these studies expressly analyzed the impact of support size on the proposed TCP variations. A logical model for High Speed TCP is inferred and altered point strategy is utilized to numerically illuminate for the use attained by contending long lasting HSTCP streams the model catches how the execution of High Speed TCP is influenced by the buffer through RTT.

The presentation of the rest of this paper is as per the following. In Section II, a model for the assessment of TCP is given and, the TCP execution is concentrated on account of a high Wth. In Section III, a Network is setup and the execution with altered supports and the technique for versatile cushion measuring are exhibited. In Section IV, the altered point method to process the use of HSTCP streams vieing for a bottleneck with a given cradle size is portrayed furthermore numerical and reproduction results are introduced; then the settled point system to register the use of HSTCP streams going after a bottleneck having RED AQM with given cushion size is depicted furthermore the numerical and recreation results are displayed.

\section{II.IMPACT OF BUFFER SIZE ON TCP START-UP}

\section{A. A Model for TCP Performance}

Consider a TCP association that exchanges documents of size $\mathrm{S}$ over a way of bottleneck transfer speed $\mu$. The broadly executed Reno rendition of TCP [6] is utilized all through the paper. Nonetheless, the investigation can be connected to alternate forms too. The system is demonstrated with a solitary bottleneck hub of rate $\mu$ and of Drop Tail Buffer of size B. T means the consistent part of the Round Trip Time (RTT) of the association (the twoway spread postponement in addition to the administration time of TCP bundles in system hubs). This is the model which has been utilized regularly as a part of the writing to study the execution of TCP $[1,4,5,7,10]$.

The characterization of TCP conduct amid the first SS stage requires the count of the window at which losses happen amid SS expecting that Wth is situated to a high esteem. This later window is known as the flood window and it is signified as WB. Alternate works expect certainly that it is equivalent to the pipe size $(B+\mu t)$. It will be demonstrated later that for a little cushion measure, this window can be free of the bottleneck transmission capacity and just a capacity of $B$ and the window build rate. Note here that all through the paper, it is expected that the recipient window is situated to a high esteem so it doesn't restrict the development of $\mathrm{W}$. Notwithstanding $\mu, \mathrm{T}$ and $\mathrm{B}$, the flood window is a capacity of the rate at which TCP expands its window amid SS. This rate is known as the forcefulness or the burstiness of SS. It is displayed with an element $\mathrm{d}$ characterized as takes as follows.

\section{- $\quad$ The aggressiveness factor $d$}

Let $\mathrm{W}(\mathrm{t})$ signify the blockage window in parcels at time $\mathrm{t}$. It is gathered that after one RTT, W is expanded amid SS by $W(t)=d$ bundles. $d$ can be the consequence of the recipient deferring Acks and sending an ACK each $d$ parcels. $d=1$ implies that the beneficiary is recognizing all the parcels and $d=2$ models the Delay ACK system broadly executed in TCP recipients. $\mathrm{d}$ can likewise display the loss of ACKs on the return way. It might likewise represent any window build strategy at the source not the same as that of standard TCP. A case is Byte Counting [1], where upon the receipt of an $\mathrm{ACK}$, the window is expanded by the quantity of recognized bundles instead of by one parcel as in standard TCP.

\section{- $\quad$ The overflow window WB}

This window is obliged to comprehend the effect of the distinctive parameters on the execution. As in $[4,10]$ a SS stage is isolated into smaller than expected cycles (MC) of length of time RTT. Let W(n) be the quantity of packets transmitted

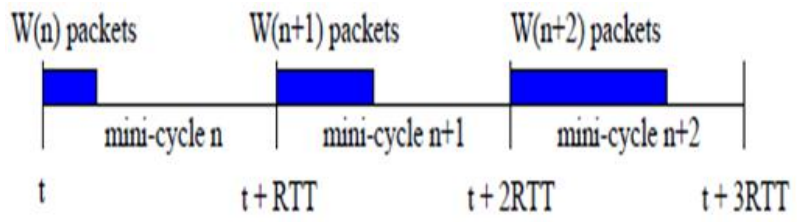

\section{Timet: Start of service of the W(n) packets}

Figure 1. Start of service of the W(n) Packets

amid MC $\mathrm{n}$. The following MC begins when the ACK for the first bundle of these W(n) packets achieves the source. As indicated by our meaning of the parameter $d$, the window size amid the following $\mathrm{MC}$ is equivalent to:

$W(n+1)=W(n)+W(n) / d=\alpha W(n)$

with $\alpha=(d+1)=d$.

Assume that the repetitive connection (1) is legitimate for each $n, 0$. Assume additionally that SS begins with a window equivalent to one parcel. Consequently,

$W(n)=\alpha^{n} W(0)=\alpha^{n}$. 
Amid SS, packets leave the bottleneck in long blasts at rate $\mu$. A blast of length $\mathrm{W}(\mathrm{n})$ is served amid MC $\mathrm{n}$ and it is trailed by an unmoving period until the entry of the blast of the accompanying MC (Figure 1). This unmoving period between blasts vanishes when the window size surpasses $\mu \mathrm{t}$. The source transmits additionally packets in long rushes because of the ACKs of packets of the past MC. Given that the quantity of packets transmitted amid a $\mathrm{MC}$ increments by a component $\alpha$, it can be gathered that the long blasts transmitted by the source have a normal rate $\alpha \mu$. Therefore, toward the start of MC $n$, the source begins to transmit a blast of length $\mathrm{W}(\mathrm{n})$ at a normal rate $\alpha \mu$. At the point when a source blast achieves the bottleneck, a line begins to develop in B at a rate $\alpha \mu-\mu=\mu / \mathrm{d}$. Here, two cases must be considered. The main case is when B doesn't contain any parcel from the past MC when the first bundle of the blast of the current MC achieves the bottleneck. The second case is the point at which a few packets from the past MC are even now holding up in B. Note here that in alternate works $[4,5,10]$, just the first case has been considered. This is right when the buffer size is little contrasted with the BDP. Nonetheless, the second case acquainted licenses us with record for all the buffer sizes. In the first case, a blast of size $\mathrm{B}(\mathrm{d}+1)$ is obliged to fill the cushion and reasons a flood. Let $n_{\tilde{0}}^{1}$ be the quantity of the MC amid which B floods. The quantity of packets transmitted amid this MC must be bigger than $\mathrm{B}(\mathrm{d}+1)$. Anyway, the quantity of packets transmitted amid the past MC must be short of what $\mathrm{B}(\mathrm{d}+1)$ generally the flood would have happened amid the past MC. Along these lines, $n \frac{1}{s}$ satisfies,

$\alpha^{n \frac{1}{B}-1}<B(d+1) \leq \alpha^{n \frac{1}{B}}$

As per our meaning of $d$, the transmission of a blast of $\mathrm{B}(\mathrm{d}+1)$ packets obliges an increment in $\mathrm{W}$ by $\mathrm{B}$ packets since the start of $\mathrm{MC} n_{B}^{1}$. It takes after that,

$$
W_{B}=W\left(n_{B}^{1}-1\right)=\alpha^{n^{1}-1}+B
$$

Presently, the second case is considered. The window size is bigger than $\mu \mathrm{t}$. The blast size needed to fill the cradle is short of what $\mathrm{B}(\mathrm{d}+1)$ since there are a few packets holding up from the past $\mathrm{MC}$. This is equivalent to the quantity of unfilled spots toward the start of the MC times $(d+1)$. The increment in the window between the start of the MC and the flood is equivalent to the quantity of void spots. Assume that the flood happens amid $\mathrm{MC} n_{B}^{2}$. At that point, $W_{B}$ gets to be equivalent to:

$$
\begin{aligned}
W_{B} & =W\left(n_{B}^{2}-1\right)+B-\left(W\left(n_{B}^{2}-1\right) \mu T\right) \\
& =B+\mu \mathrm{T}
\end{aligned}
$$

Two declarations for $\mathrm{W}_{\mathrm{B}}$ are then accessible. On the chance that the window size amid MC $n_{B}^{1}-1$ is short of what $\mu \mathrm{t}$, then $\mathrm{W}_{\mathrm{B}}$ will be given by mathematical statement (2), else it will be given by comparison (3). These two representations can be joined into a solitary one as said in the accompanying hypothesis.

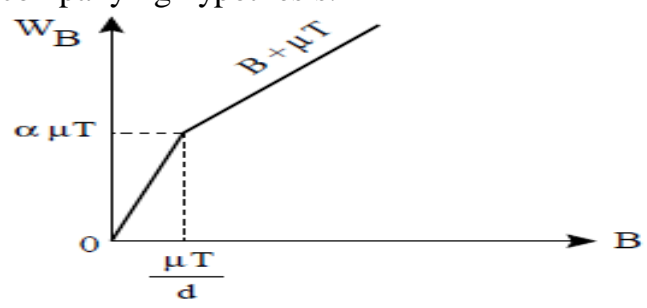

Figure 2. The overflow Window $W_{z}$ vs. B

Theorem 1: In the event that SS is not ended before the event of misfortunes, the cushion at the entrance of the bottleneck connection will flood at a window:

$$
W_{B}=B+\min \left(\mu T, \alpha^{n_{B}-1}\right),
$$

\section{B. Impact of High $W_{\text {thi }}$ on Performance}

Here, the situation where Wth is situated higher than WB and where TCP uses its SS calculation to gage the system limit is considered. Misfortunes happen and the execution is a capacity of $w_{\mathrm{z}}^{r}$, the new system limit evaluate after the recuperation from misfortunes.

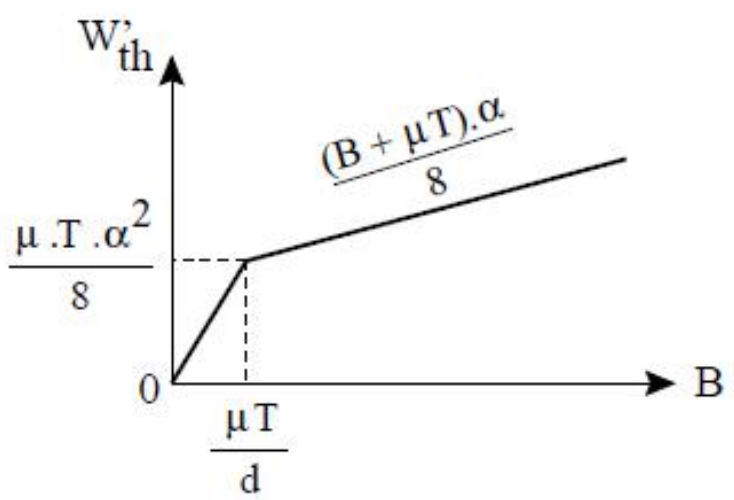

Fig 3. The new capacity estimate vs. B

\section{- Calculation of $\boldsymbol{W}_{t \pi}^{r}$ :}

The buffer flood is caught one RTT after its event. Amid this RTT, W increments by awb unless the source gets in CA. This is the situation when $\mathrm{WB}<\mathrm{Wth}<\alpha \mathrm{wb}$. Blockage is located at a window WD equivalent to:

$W_{\mathrm{D}}=\min \left(\mathrm{W}_{\mathrm{th}}, \alpha W_{\mathrm{B}}\right)$

Here, TCP sets $\mathrm{W}$ and $\mathrm{W}_{\text {th }}$ to half $\mathrm{W}_{\mathrm{D}}$ and begins to recoup from misfortunes. Frequently, it succeeds to locate the initial two misfortunes through Duplicate Acks. The third and the ensuing misfortunes require a Timeout to be recognized [6]. Reno partitions its window by two upon each misfortune recognition. Hence, in the greater part of the cases, the SS limit after the Timeout is situated to one eighth WD. It is situated to one fourth WD when the second misfortune can't be recognized through Duplicate Acks. In the continuation, it is accepted that $W_{\text {th }}^{f}=W_{D}=8$. 
It is similarly expected that Wth is situated much higher than $\alpha w b$ so that the is constantly caught at WD $=\alpha w b$.

\section{- Impact of $B$ and $d$ on Performance:}

Utilizing the line as a part of Figure 2, Figure 3 is plotted, the variety of $W_{i}^{r}$ It is well seen that the evaluation gave by SS moves to zero when the cushion size does. Above $\mu \mathrm{t}=\mathrm{d}$, the reduction in execution is created by the diminishing in the channel measure however the forcefulness of TCP amid SS has no effect on the evaluation. Nonetheless, underneath $\mu \mathrm{t}=\mathrm{d}$, the cradle gets to be not able to assimilate the bursty activity of TCP and the blockage shows up before arriving at the funnel size. An increment in TCP forcefulness for this situation diminishes the appraisal and may break down the execution as opposed to enhancing it. It is concentrated on in the accompanying, this communication between cradle size and TCP forcefulness. Two cases are viewed as: First it is assumed that the forcefulness is controlled at the collector which recognizes each $d$ information packets. For this situation, both SS and CA are

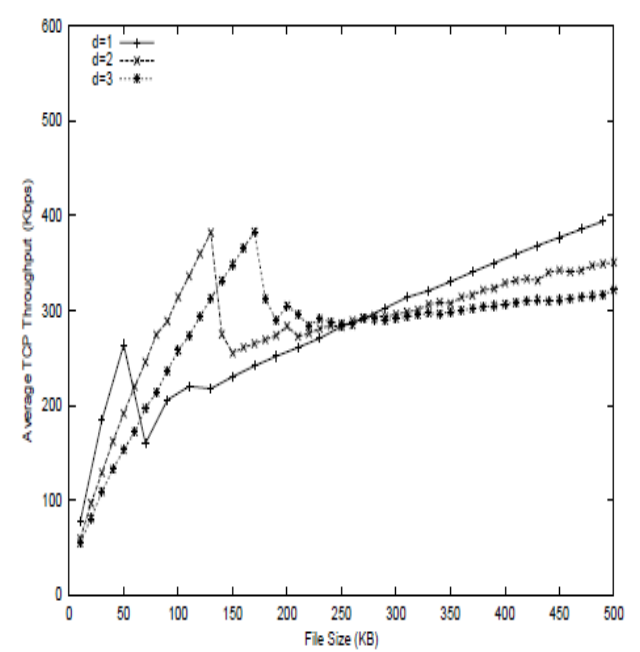

Figure 4. Throughput vs. file size for $B=20$ packets

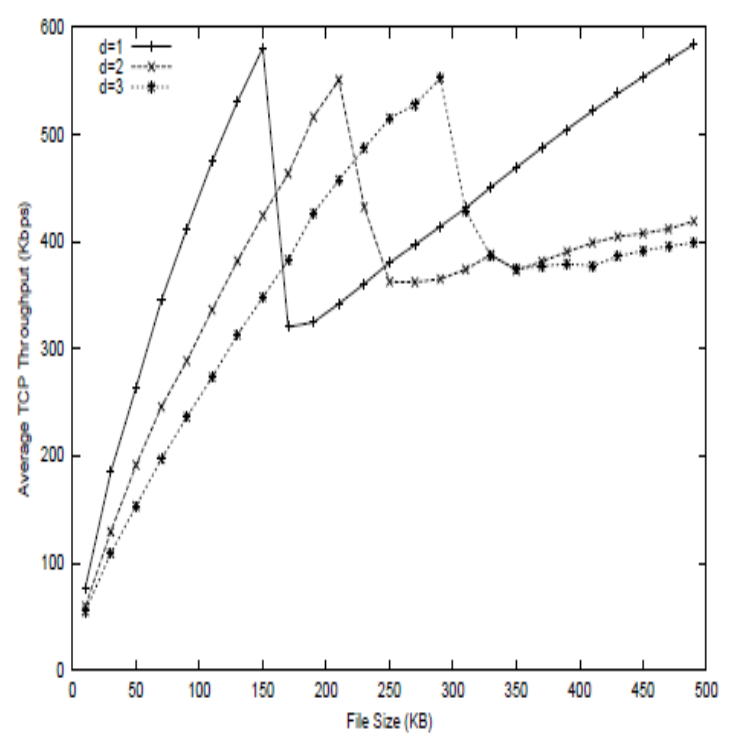

Figure 5. Throughput vs. file size for $B=70$ Packets influenced. Second, the situation where the forcefulness is controlled at the source is considered. During this situation, the source can recognize SS and CA and can accordingly receive diverse elements $\mathrm{d}$ amid each stage.

A receiver-controlled $d$ : As seen from Figure 3, if there should be an occurrence of a B littler than $\mu \mathrm{t}=\mathrm{d}$, an increment in $\mathrm{d}$ enhances the system limit assess after the Timeout. This ought to prompt a change in the execution. Be that as it may, on account of a collector controlled d, the increment in $\mathrm{d}$ abates likewise the window development amid CA. The increase attained in $w_{t h}^{r}$ when expanding $\mathrm{d}$ will be repaid later by the slower window development amid CA. The execution begins in order to break down after a little change. This conduct is seen in Figures 4 and 5 where it has been plot for two diverse B, 20 packets and 70 packets, the throughput as a capacity of the record size. Three estimations of $d$ are considered. For a given $\mathrm{d}$, it is perceived that toward the starting, the throughput increments rapidly with the record size. Little records are exchanged totally amid the SS stage and the quick increment in the throughput is because of the quick window increment amid SS.

For every d, a descending bounce in the execution for some document size can be seen. This relates to the presence of misfortunes amid the SS stage. After this bounce, the source gets in CA bringing about a slower increment in the throughput. The more distant the source gets in CA, the littler is the influence of the first SS stage. After this jump, the source gets in CA resulting in a slower increase in the throughput. The farther the source gets in CA, the smaller is the impact of the first SS phase.

The normal throughput ought to keep expanding in an asymptotic way until the normal throughput in the unfaltering is arrived at. Typically, the greatest normal throughput or the most extreme usage of system assets is a capacity of $\mathrm{B}, \mu$ and $\mathrm{T}$ and not the forcefulness. The forcefulness decides the rate at which it unites to the consistent state. A little $\mathrm{d}$ is quite greater to an extensive one at whatever point the document is exchanged without misfortunes. Once these misfortunes happen for the little $\mathrm{d}$ (the more forceful form), the substantial $\mathrm{d}$ begins to give better execution on the grounds that it keeps away from the Timeout. This will keep on proceeding until misfortunes happen for the substantial $\mathrm{d}$ too. In the event of little supports, regardless of the possibility that misfortunes show up for the extensive d, it keeps on going preferred execution over the little $\mathrm{d}$ for specific records sizes because of the higher system limit gauge given by it. Be that as it may, the CA stage of it is excessively moderate so it loses this benefit later. In the event of expansive cradles nonetheless, the lessening of the forcefulness doesn't enhance the appraisal. Along these lines, the execution of a huge $d$ gets to be more awful than a little $d$ when misfortunes happen. In this way, a collector controlled d influences both SS and CA. The most minimal conceivable $\mathrm{d}$ must be utilized amid CA to ensure a quick meeting to the relentless state. The most generally utilized component is $\mathrm{d}=2$ (Delay ACK instrument). Amid SS, $d$ must be picked in such an approach to build the window as fast as would be prudent to the fill the funnel. The best execution 
is gotten when it is begun by a little $d$ then changed to a bigger one equitable before the flood of cushion and it is proceeded like this until the channel size is arrived at. This comprises in lessening SS burstiness with the increment in the blockage window. Such component is hard to execute given that TCP is not mindful of the cradle estimate in system hubs. In any case, this provides for us a rule on the best way to change the element $d$ on ways with little buffers.

A sender-controlled $d$ : In this segment the effect of a change in the window expand rate amid SS on the execution is contemplated. The window expand rate amid CA is kept unaltered. This allows an end of the effect of $d$ on the CA stage. Such conduct obliges a change at the source on the grounds that it is the main component ready to recognize the two stages. It is expected in the accompanying that the collector

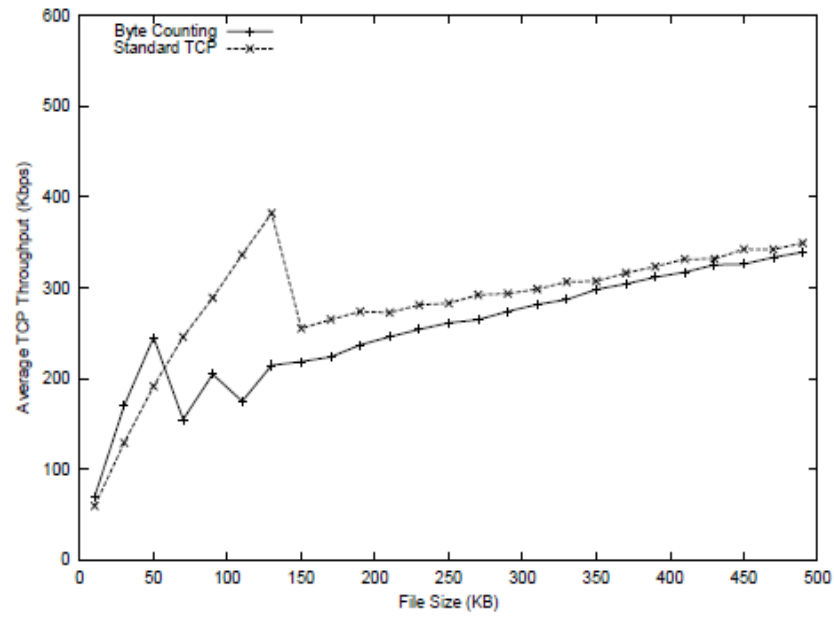

Figure 6. Throughput vs. file size for $B=20$ packets

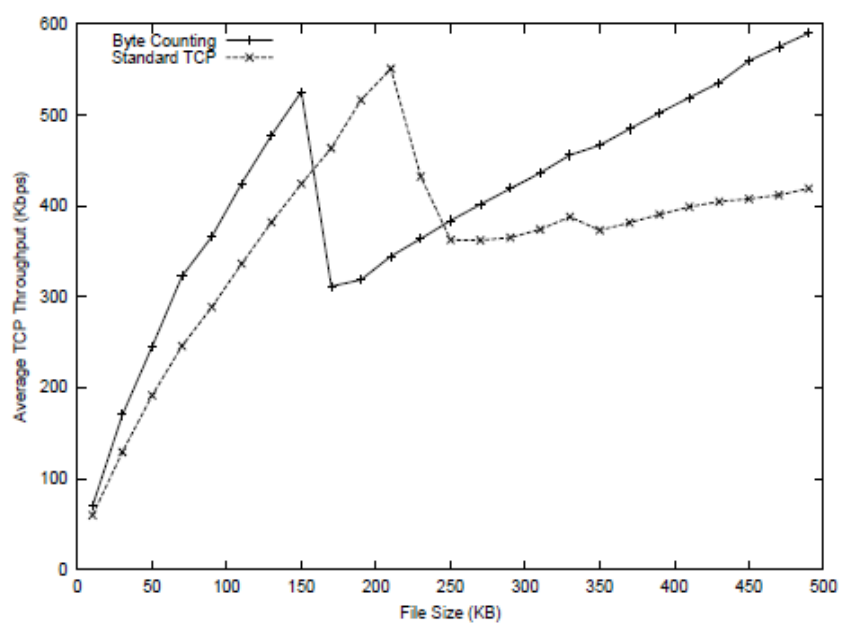

Figure 7. Throughput vs. file size for $B=20$ packets

is recognizing each other information parcel and that the source executes standard TCP amid CA. Changing the variable $d$ at the source can be fulfilled by changing the measure of the window increment upon the receipt of an ACK. Given that Acks are postponed at the objective, standard TCP compares to $\mathrm{d}=2$. Byte Counting [1] relates to $\mathrm{d}=1$. Changing $\mathrm{d}$ from 2 to 1 has been demonstrated to enhance the execution $[1,3]$. Our investigation demonstrates that this can be the situation on ways with substantial supports. Then again, on ways with little cradles, expanding the forcefulness decreases the system limit appraise and decays the execution. Figures 6 and 7 clarify this issue. These figures demonstrate the throughput as a capacity of the record size for the two cradles 20 parcel and 70 packets. In each one figure, one of the two lines speaks to standard TCP and the other line speaks to Byte Counting. For an expansive cradle, Byte Counting works superbly and gives better execution. On the other hand, for a little cradle, Byte Counting is aggressive to the point that it fills the cushion before filling the channel. This gives lower appraisal and consequently lower execution for the vast majority of the document sizes.

\section{BUFFER SIZING FOR TCP FLOWS}

\section{A. Network Setup}

The situations where the Access Point (AP) goes about as a remote switch between the WLAN and the wired Internet are considered. Transfer streams are from remote stations in the WLAN to server(s) in the Internet, while downloads are from wired server(s) to stations in the WLAN. At the MAC layer, IEEE 802.11g parameters are utilized as indicated as a part of Table I. The transfer speed between the AP and server(s) is $100 \mathrm{Mbps}$. TCP Reno with SACK is utilized. It is noted that in Wlans, TCP ACK packets without any prioritization can be effortlessly lined/dropped because of the way that the essential 802.11 DCF guarantees that stations win a generally equivalent number of transmission opportunities. In point case consider $n$ stations each one convey one TCP transfer stream. The TCP Acks are transmitted by the AP. While the information packets for the $n$ streams have a total $n /(n$ +1 ) offer of the transmission opportunities the TCP Acks for the $n$ streams have just a $1 /(n+1)$ offer. Issues of this sort are referred to debase TCP execution altogether as lining and dropping of TCP Acks upset the TCP ACK timing system. Taking after [3], this issue is tended to utilizing 802.11e. At the AP and each one station, TCP Acks are dealt with as a different activity class, gathering them into a line which is allocated high need through Cwmin $=3$, Cwmax $=7$, AIFS $=6$. This makes use of 2 out of the 4 available queues in 802.11e.

TABLE-I

MAC AND PHY PARAMETERS

\begin{tabular}{|l|c|}
\hline$T_{\text {sIFs }}(\boldsymbol{\mu})$ & 10 \\
\hline Idle slot duration & 9 \\
\hline Retry limit & 11 \\
\hline Packet size (bytes) & 1000 \\
\hline PHY data rate (Mbps) & 54 \\
\hline PHY basic rate (Mbps) & 6 \\
\hline PLCP rate (Mbps) & 6 \\
\hline
\end{tabular}



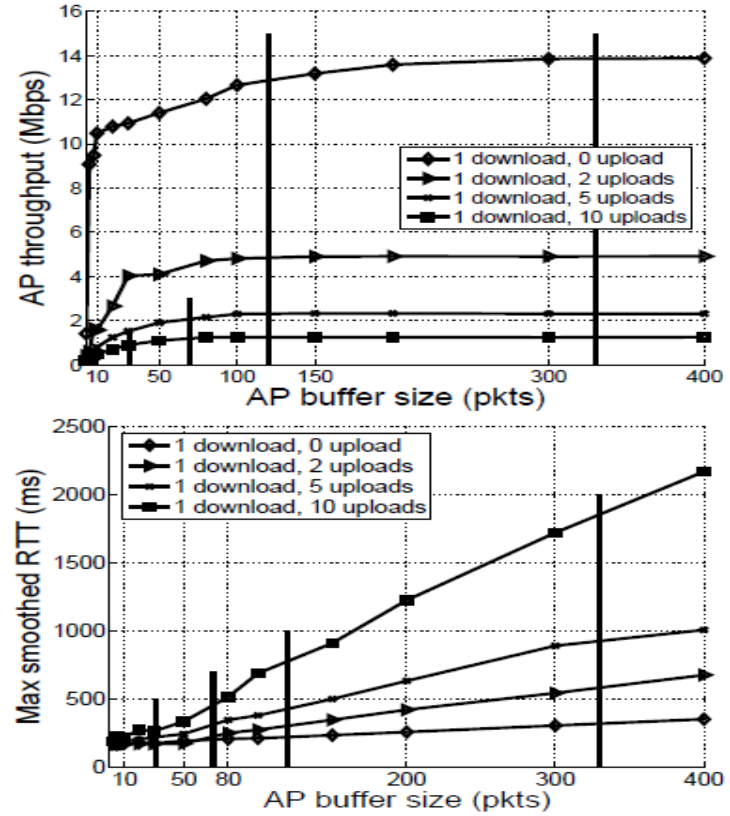

Figure 8. Performance with fixed buffer sizes. Data shown for 1 download flow and 0, 2, 5, 10 uploads - corresponding BDP values marked by vertical lines. One upload flow per wireless station. Max smoothed RTT denotes the maximum TCP srtt value observed. Wired backhaul link bandwidth 100Mbps, RTT 200ms.

\section{B. Performance with Fixed Buffers}

Rather than wired systems, the mean administration rate at a remote station is not settled yet rather relies on the level of channel discord and the system load. This is represented in Fig. 1 where the throughput and postponement of a download stream are plotted as a capacity of the AP support size when the quantity of contending transfer streams (with one transfer stream every remote station) is differed. So also to wired systems, the throughput dependably increments monotonically with the support size, arriving at a most extreme over a limit cushion size. It can likewise be seen that the download throughput falls as the quantity of contending transfers increments. The variety in throughput can be significant, e.g., the greatest throughput transforms from $14 \mathrm{mbps}$ to $1.25 \mathrm{mbps}$ as the quantity of contending transfers transforms from 0 to 10 . Thus, the BDP - checked by vertical lines in Fig. 1 - additionally changes fundamentally and this is reflected in buffering prerequisites. For instance, it can be seen from Fig. 1 that with no contending transfers the limit cradle measure above which the AP attains most extreme throughput is 338 packets, while for 10 contending transfers this cushion size tumbles to give or take 70 packets. In perspective of this conduct, one conceivable methodology is to size supports focused around most detrimental possibility conditions, i.e., focused mainly around the specific conditions obliging the biggest buffering to attain high throughput. Notwithstanding, while guaranteeing high throughput, this takes on at the

expense of high dormancy. For instance, it can be seen from Fig. 1 that when an altered cushion size of 338 packets is utilized (which as a part of this illustration guarantees greatest throughput paying little heed to the quantity of battling stations), the round outing inertness experienced by the download stream is around $300 \mathrm{~ms}$ with no transfers yet climbs to around $2 \mathrm{~s}$ with 10 fighting transfer stations. This happens in the absence of the fact that TCP's blockage control calculation tests for transmission capacity until bundle misfortune happens thus download streams will have a tendency to fill supports with any sizes. In addition, the mean lining deferral of a support of size $\mathrm{Q}$ with mean administration rate $\mathrm{B}$ is $\mathrm{Q} / \mathrm{B}$. Consequently, the lining postponement at the AP relies on upon the administration rate, which thusly relies on upon the quantity of battling remote stations and their offered burden. For a settled size support, an abatement in the administration rate of a component $b$. On the other side, estimation of the buffer to attain lower inactivity over all system conditions has a go at the expense of decreased throughput, e.g., a support size of 30 packets guarantees inertness of $200-300 \mathrm{~ms}$ for up to 10 fighting transfer stations yet when there are no battling transfers the throughput of a download stream is just around $75 \%$ of the greatest achievable.

Notwithstanding varieties in the mean administration rate, it is additionally noted that the arbitrary nature of 802.11 operations prompts brief time-scale stochastic variances in administration rate. This is generally not quite the same as wired systems and straightforwardly effects buffering conduct. Stochastic vacillations in administration rate can prompt early line flood and lessened connection usage. Case in point, from Fig. 1 with 10 transfers the most extreme download throughput is $1.25 \mathrm{mbps}$, yielding a BDP of 31 packets. On the other side, it can be observed that at this support estimate the accomplished download throughput is just around $60 \%$ of the most extreme - a cushion size of no less than 70 packets is obliged to attain $100 \%$ throughput. The stochastic variances in administration rate lead to a necessity to build the cradle measure over the BDP with a specific end goal to suit the effect of these changes. The measure of over-provisioning obliged may be limited utilizing measurable contentions, yet it is not sought after further here because of space obligations. It is to be noted that, then again, that a basic however compelling methodology is to over-procurement by a settled number of packets over the BDP. For instance, from Fig. 1 it can be seen that over-provisioning by 40 packets is sufficient for the scope of conditions considered, and it is observed that this methodology meets expectations all the more for the most.

\section{Adaptive Buffer Sizing}

Roused by the previous perceptions and the trouble of selecting a settled cushion size suited to a scope of system conditions, the consideration of the utilization of a versatile support estimating procedure is done. It is noted that a remote station can promptly measure its own administration rate by perception of the between administration time, i.e., the time between bundles touching base at the leader of the system interface line ts and being effectively transmitted te (which is demonstrated by getting accurately the relating MAC ACK.). Note that 
this estimation can be promptly executed in genuine gadgets and causes minor calculation load. Let $\operatorname{Tserv}(\mathrm{t})$ be the between administration time at time $t$, exponential smoothing is utilized to figure the mean between administration time Tserv $=\alpha$ tserv $+(1-\alpha)$ (te - ts $)$ where $\alpha=0.999$

Utilizing this estimation the accompanying versatile procedure is proposed. Let $\mathrm{T}$ be the target lining delay 2 . The cradle size $\mathrm{Q}$ is chosen as per $\mathrm{Q}=\min (\mathrm{t} / \mathrm{T}$ serv, $\mathrm{qmax})$ where Qmax is situated to be 400 bundles. This will diminish the support size when the administration rate falls and expand the cradle size when the administration rate climbs, in order to keep up a roughly consistent lining postponement of $\mathrm{T}$ seconds. This viably controls the support size to stay equivalent to the BDP as the mean administration rate differs. To record for the effect of the stochastic nature of the administration rate on cushion size necessities, this upgrade guideline is adjusted to $\mathrm{Q}=$ $\min (\mathrm{t} / \mathrm{Tserv}+\mathrm{a}, \mathrm{qmax})$ is an over-provisioning add up to suit fleeting vacillations in administration rate.

Taking into account the estimations in Fig. 1 and others, it has been discovered that a quality equivalent to 40 parcels functions admirably over an extensive variety of system conditions. The viability of this straightforward versatile calculation is delineated in Fig. 2. Here the plot is given for throughput rate and smoothed RTT of download streams as the quantity of download and transfer streams is differed. It can be seen that the versatile calculation keeps up high throughput productivity over the whole scope of working conditions. This is accomplished while keeping up the inertness roughly steady at around $400 \mathrm{~ms}$ - the idleness climbs somewhat with the quantity of transfers because of the over-provisioning a to suit stochastic variances in administration rate. Fig. 3 exhibits the capacity of the versatile algorithm to react rapidly to changing system conditions. At time 200s the quantity of transfers is expanded from 0 to 10 . It can be seen that the cradle measure rapidly adjusts to the changed conditions while keeping up high throughput product.

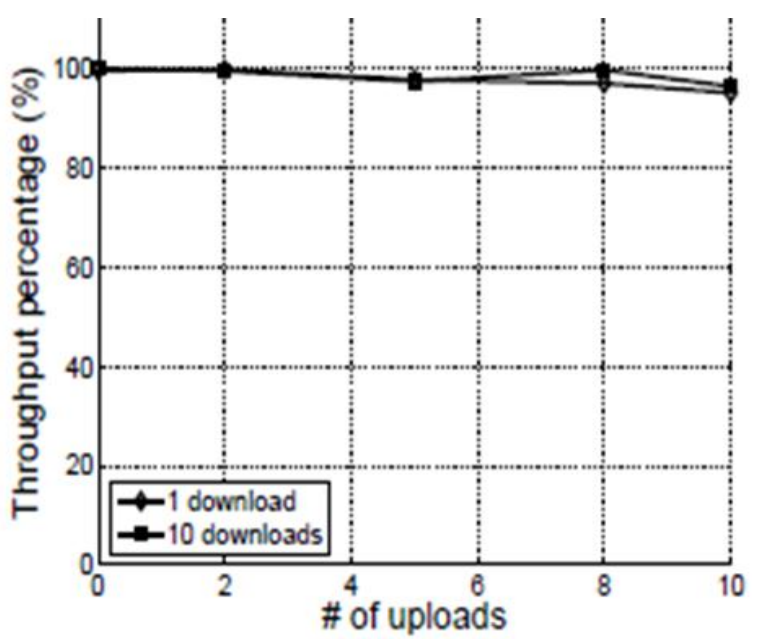

Figure 9. Throughput Percentage

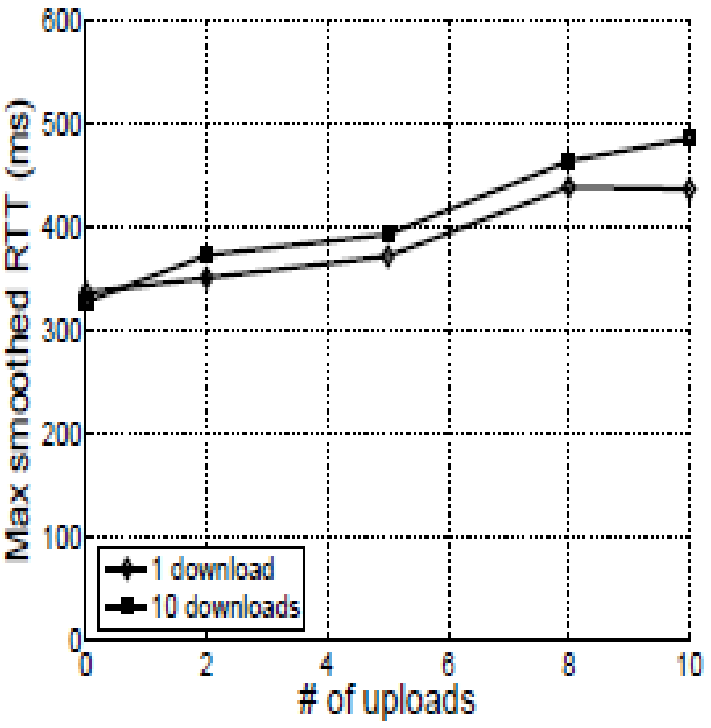

Figure 10. Max Smoother RTT

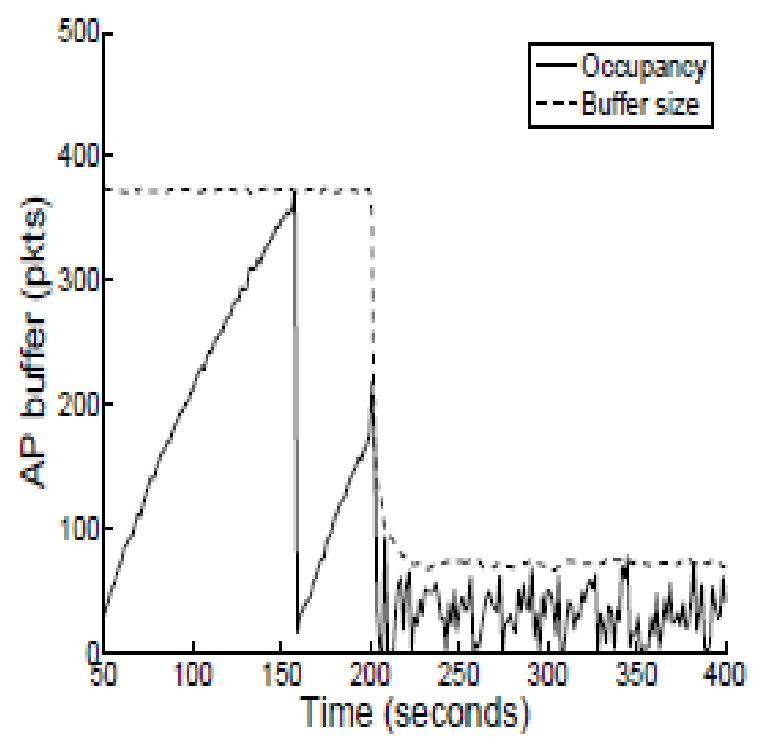

Figure 11. AP Buffer

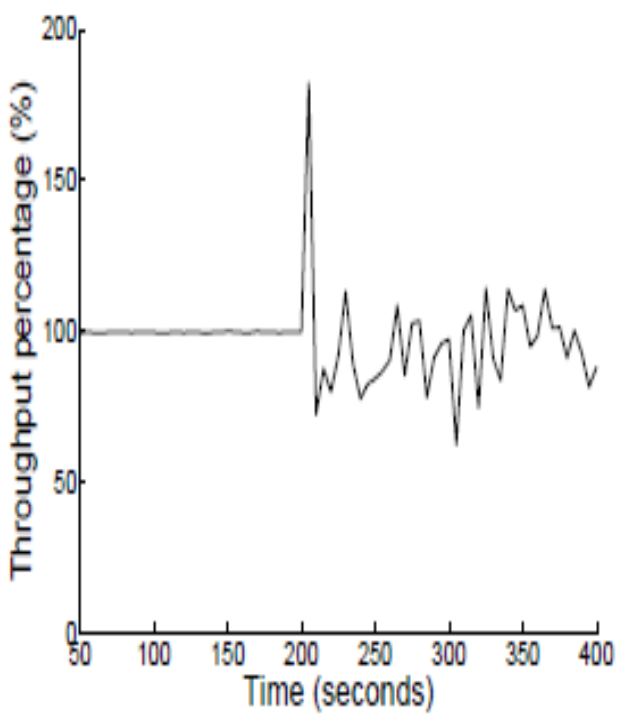

Figure 12. Throughput Percentage 


\section{INFLUENCE OF ROUTER BUFFER SIZE ON HIGH SPEED TCP}

\section{A. Fixed Point Approximation with Drop Tail}

In this area, the altered point iterative technique to assess HSTCP accepting a $\mathrm{M} / \mathrm{M} / 1 / \mathrm{K}$ model of the bottleneck line is depicted. The heap on the bottleneck connection is given by:

$$
\rho=\frac{1}{\mathrm{c}} \sum_{i=1}^{\mathbb{N}} \lambda_{i}\left(p, R T T_{i}\right)
$$

From established $\mathrm{M} / \mathrm{M} / 1 / \mathrm{K}$ results:

$$
\rho=\frac{\rho^{R}(1-\rho)}{1-\rho^{R+1}}: R_{l}=2 d_{i}+\frac{M S S}{C} \frac{\rho}{1-\rho}-\frac{R \rho^{R}}{1-\rho^{R}}
$$

where $d_{i}$ is the proliferation deferral of the ii-th association and MSS the most extreme portion size. So the heap can be gotten from Eq. (4):

$\rho=\frac{1}{C} \sum_{i=1}^{\mathbb{N}} \lambda_{i} \frac{\rho^{n}(1-p)}{1-p^{R+1}} \cdot 2 d_{i}+\frac{M S S}{C} \frac{\rho}{1-\rho}-\frac{K p^{K}}{1-\rho^{K}}$

It is watched the right hand side of Equation (5) is consistent in $\rho \in[0,1]$. The throughput, $\lambda$, of HSTCP is constant in $\mathrm{p}$ pand $\mathrm{p}$ pis persistent in $\rho$ Therefore, in any event there exists an altered point for Eq. (5). The estimation of the subsidiary of the iterative capacity relies on upon the cushion size and consequently, the uniqueness of the altered purpose of the capacity relies on upon the buffer.

Evaluation: The impact of cushion sizes on the usage accomplished by 10 perpetual High Speed TCP associations beginning at diverse times is reenacted. The topology is demonstrated in Figure 13. The normal connection, R1-R2 R2is 1gbps with 50ms deferral. The Rtts of the associations are diverse extending from $115.5 \mathrm{~ms}$ to $124.5 \mathrm{~ms}$ and normal RTT is $120 \mathrm{~ms}$. The cushion size at R1-R2is fluctuated as a small amount of $125001 \mathrm{~kb}$-parcels ( $80 \%$ of BDP of the biggest RTT.) The BDP of just

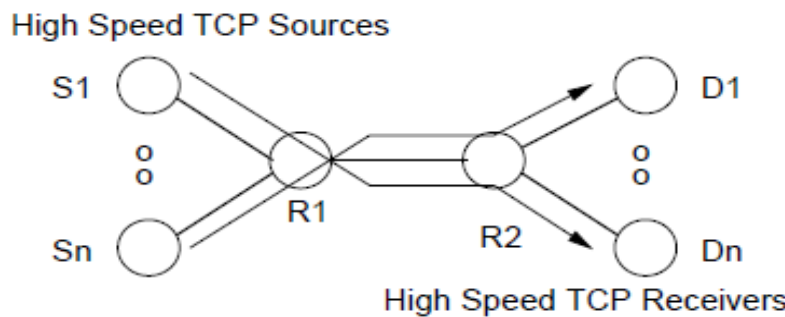

Figure 13. Simulation Topology

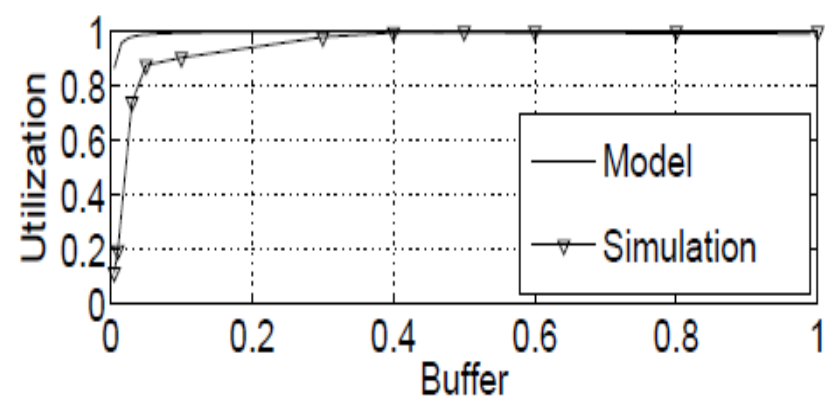

Figure 14. Throughput as function of Buffer size fraction ( $\max 12500)$

R1-R2 is 12500 bundles. In the investigations, the estimations of $\omega_{h}=83000, \omega_{l}=38, p_{h}=10^{-7}$ and $p_{l}=10^{-3}$ have been used. Observing the bottleneck utilization as a function of buffer size in Figure 14, it is found that results from simulations and our analytical model are quite close. In simulations, it is found that the loss rate is mostly equal to $p_{l}$. It is observed that buffer size equal to $10 \%$ of the bandwidth-delay product can sustain more than $90 \%$ utilization. The discrepancy between the two curves in Figure 14 mainly arises from our $\mathrm{M} / \mathrm{M} / 1 / \mathrm{K}$ assumption and simplifying approximations that have been made in deriving the closed form throughput expression. In the next experiment, the bandwidth of the common link R1-R2 is changed to $2.5 \mathrm{Gbps}$ and delay to $10 \mathrm{~ms}$. The RTTs of the connections are different ranging from $38 \mathrm{~ms}$ to $142 \mathrm{~ms}$ and average RTT is $90 \mathrm{~ms}$. The buffer size is varied as a fraction of $62501 \mathrm{~KB}$-packets (35\% of BDP of largest RTT connection.) The BDP of just R1-R2 is 6250 packets. The window and queue evolution of two competing High Speed TCP flows in case of under-buffered and overbuffered (Figure 15) bottleneck router using the topology shown in Figure 13 are plotted. The RTT of HSTCP1 is almost twice that of HSTCP2. The bottleneck bandwidth is $1 \mathrm{Gbps}$ with $10 \mathrm{~ms}$ of delay. The unfairness evident from the window dynamics is due to difference in RTTs of the two connections. It also seen that a larger bottleneck buffer reduces the unfairness between two connections. Thus, one should consider the effect of buffer size on the tradeoff between RTT fairness and utilization.

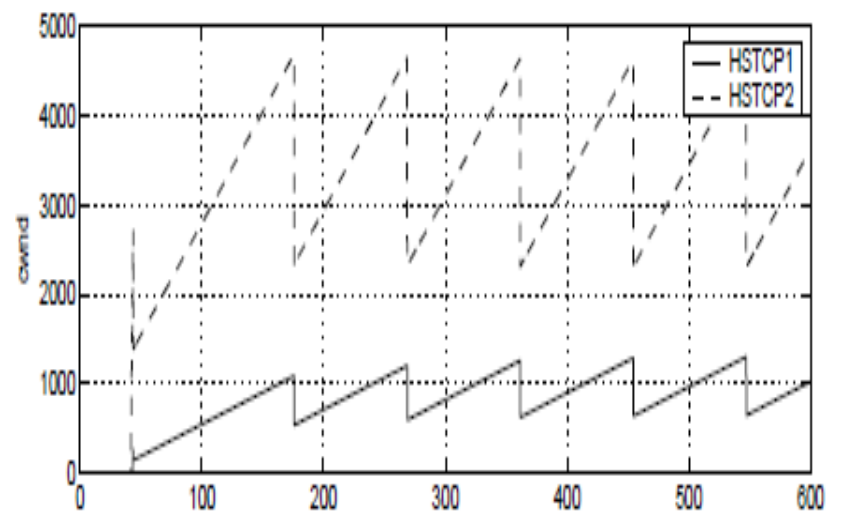



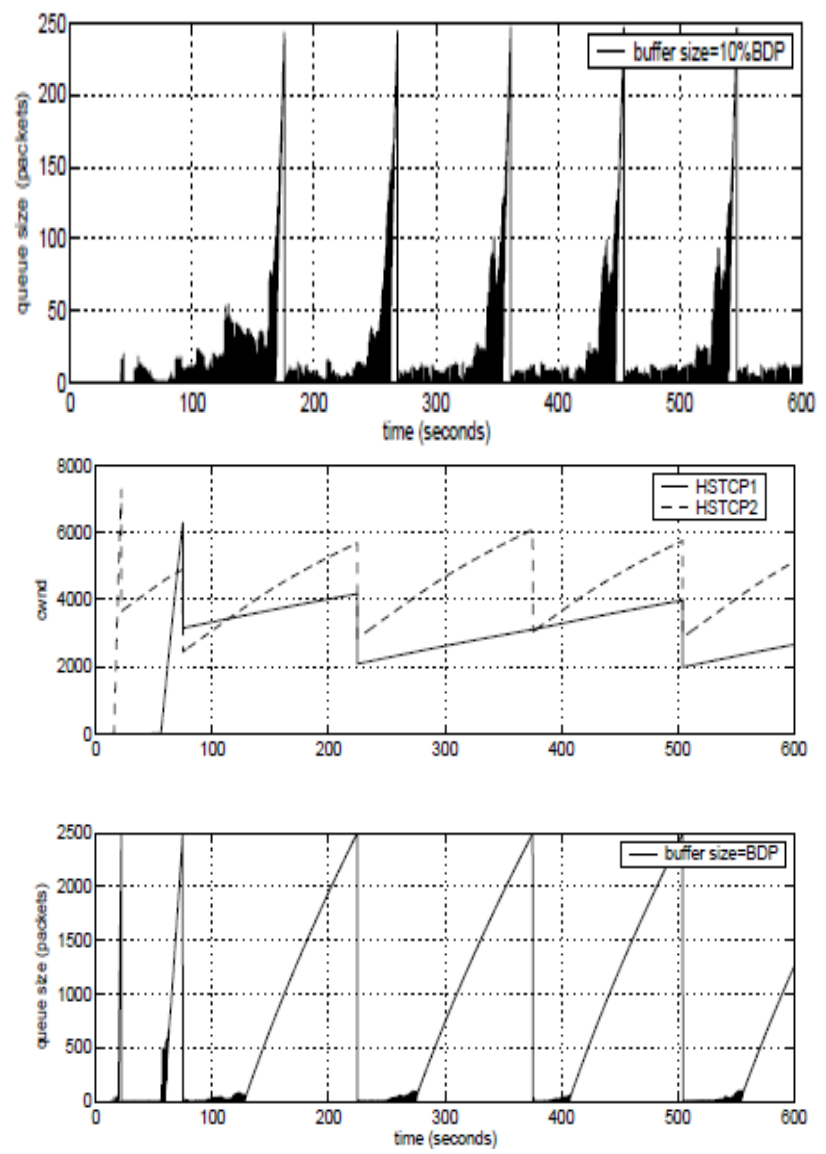

Figure 15. Window behavior of two High Speed TCPs and drop tail queue size in over-buffered case

\section{Fixed Point Approximation with RED AQM}

In this area, the execution of High Speed TCP under RED is assessed. The investigative model of RED is taken after. RED aides in uprooting the predisposition against bursty streams and blast sizes are extensive on account of High Speed TCP streams. Utilizing the PASTA property, the drop likelihood of a bundle at a RED line is given by:

$$
\begin{aligned}
& P_{\text {red }}=\sum_{n=0}^{\pi} \pi(n) p(n) \\
& =0 \\
& =\frac{\left(n-m i m_{t h}\right) p_{\max }}{\max x_{\text {th }}-m n_{\mathrm{th}}} \\
& \text { if } n \leq m m_{t h} \\
& \text { else }
\end{aligned}
$$

Evaluation: The impact of support size on the usage accomplished by 10 perpetual High Speed TCP associations under RED is reenacted. The normal connection R1-R2 2is $1.0 \mathrm{gbps}$ with $50 \mathrm{~ms}$ postponement. The cradle size is differed as a small amount of $125001 \mathrm{~kb}-$ bundles. The associations begin at distinctive times furthermore their Rtts are diverse going from $115.5 \mathrm{~ms}$ to $124.5 \mathrm{~ms}$ and normal RTT is $120 \mathrm{~ms}$. Versatile RED is utilized as a part of which $\min _{\mathrm{th}}$ and $\max _{\mathrm{th}}$ are powerfully balanced. In our numerlical assessment basic RED is utilized for numerical tractability; $m m_{t h}=40 \%$ of full support size and $\max _{z h}$ to full cushion size are situated. The use of High Speed TCP in the vicinity of RED is plotted in Figure 16. The misfortune rate is $10-7$ for cradle degree $\geq 0.1$

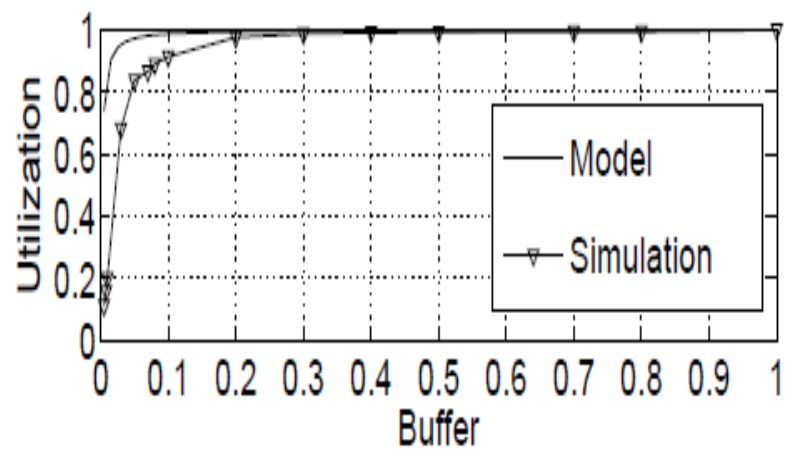

Figure 16. Throughput as function of buffer size, $\operatorname{mi} \boldsymbol{m}_{\mathrm{z}_{2}}=0.4 \mathrm{xmin}$ (BDP, buffer size), $\max _{t_{2}}=\min (\mathrm{BDP}$, buffer size), $\mathrm{N}=10, \mathrm{C}=1 \mathrm{Gbps}$

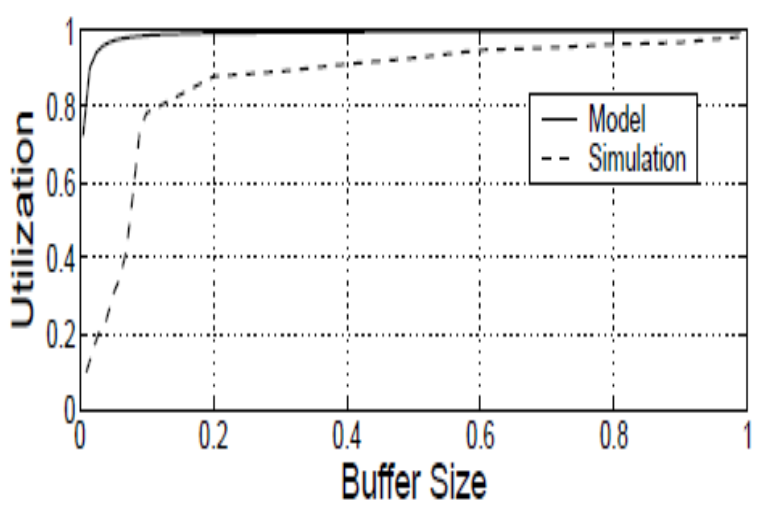

Figure 17. Throughput as function of buffer size, $m i n_{\mathrm{s}}=0.4 \mathrm{xmin}$ (BDP, buffer size), $\max _{\mathrm{t}}=\min (\mathrm{BDP}$, buffer size), $\mathrm{N}=10, \mathrm{C}=2.5 \mathrm{Gbps}$

A comparable investigation is plotted in Figure 16 where the limit is changed to $2.5 \mathrm{gbps}$ and postponement to $10 \mathrm{~ms}$. The Rtts are diverse running from $38 \mathrm{~ms}$ to $142 \mathrm{~ms}$ and normal RTT is $90 \mathrm{~ms}$. The cushion size is differed as a small amount of 62501 kbpackets. It is observed that the misfortune rate is around 10-6. In spite of utilizing Adaptive-RED in the reenactments, close concurrence with the numericals.

\section{CONCLUSION}

In this paper, the conduct of TCP amid the first SS stage has been concentrated on. This allowed us to assess the execution of shorts exchanges and the viability of the suggestions made to enhance this execution. It is discovered that, on ways with little cushions contrasted with their BDP, the blockage amid SS may seem ahead of schedule before filling the funnel size. Subsequently, the worth to provide for Wth toward the start of the association, keeping in mind the end goal to dodge misfortunes must record for the cushion measure overall misfortunes may not be dodged. Additionally, for this situation, it is discovered that any increment in TCP forcefulness falls apart the execution as opposed to enhancing it. A rule for how to expand the window in these ways is displayed. 
Next, the assignment of measuring cradles is considered for TCP streams in 802.11e Wlans. Various essential new issues emerge contrasted with wired systems, including the way that the mean administration rate is reliant on the level of channel conflict and parcel between administration times change stochastically because of the arbitrary nature of CSMA/CA operation. Propelled by these perceptions, a versatile support measuring calculation is proposed which imitates the traditional BDP lead and exhibits its viability through recreations. Support estimating while rate adjustment is empowered is left as future work, in spite of the fact that it is accepted that the proposed calculation will work. Future work likewise incorporates thought of the likelihood of diminishing cushion sizes when multiplexing happens [10].

From our numerical results obtained utilizing the altered point strategy and also from reproductions, it is presumed that regularly, an industrious High Speed TCP association crossing a bottleneck join has a low sending rate when the cushion size is little. The throughput increases as cradle size increases. It is found that a cushion size of $10 \%$ of the data transmission delay item can keep up no less than $90 \%$ usage. Expanding the support estimate past $10 \%$ builds the use barely arriving at very nearly $98 \%$ when the cushion size is around $20 \%$ of the data transfer capacity delay item. Then again, a little cushion measure below $10 \%$ of the transmission capacity delay item causes numerous bundle drops, which prompt an uncommon drop in the use and may decrease High Speed TCP to consistent/standard TCP. It is noted that our results are predictable with a late free study, which demonstrates that the general guideline of setting the support size equivalent to the transfer speed deferral item is difficult. The results are introduced considering a solitary bottleneck. Then again it is accepted that cradle prerequisites will have comparable patterns on account of numerous congested bottlenecks. However one needs to accept that, inspite of the fact that it has just been centered around long lasting streams, it

will be fascinating to explore the execution of brief streams and consistent rate streams (e.g. UDP) on rapid connections with distinctive cushion sizes.

In our future work, it is proposed to explore the impacts of support size on the execution of other rapid TCP variations, e.g. Quick TCP [21] which transform its window as per cushion delay.

\section{REFERENCES}

[1] M. Allman, "On the Generation and Use of TCP Acknowledgments", Computer Communication Review, Oct. 1998.

[2] M. Allman, S. Floyd, and C. Partridge, “Increasing TCP's Initial Window", RFC 2414, Sep. 1998.

[3] M. Allman, D. Glover, and L. Sanchez, "Enhancing TCP Over Satellite Channels using Standard Mechanisms", RFC 2488, Jan. 1999.

[4] Altman, J. Bolot, P. Nain, D. Elouadghiri, M. Erramdani, P. Brown, and D. Collange, "Performance Modeling of TCP/IP in a Wide-Area Network", $34^{\text {th }}$ IEEE Conference on Decision and Control, Dec. 1995.

[5] Barakat, N. Chaher, W. Dabbous, and E. Altman, "Improving TCP/IP over Geostationary Satellite Links", IEEE Globecom, Dec. 1999.

[6] K. Fall and S. Floyd, "Simulation-based Comparisons of Tahoe, Reno, and SACK TCP", Computer Communication Review, Jul. 1996.

[7] Kumar, "Comparative Performance Analysis of Versions of TCP in a Local Network with a Lossy Link", IEEE/ACM Transactions on Networking, Aug. 1998.

[8] J. Hoe, "Improving the Start-up Behavior of a Congestion Control Scheme for TCP", ACM Sigcomm, Aug. 1996.

[9] V. Jacobson, "Congestion avoidance and control", ACM Sigcomm, Aug. 1988

[10] G. Appenzeller, I. Keslassy, and N. McKeown,"Sizing router buffers," Proc. ACM SIGCOMM, 2004.

[11] Chatfield, The Analysis of Time Series, An Introduction, CRC Press 2004.

[12] J. Leith, P. Clifford, D. Malone, and A. Ng, "TCP Fairness in 802.11e WLANs," IEEE Communications Letters, vol. 9, no. 11, pp 964- 966, 2005.

[13] Malone, P. Clifford, and D. J. Leith, "On Buffer Sizing for Voice in 802.11 WLANs," IEEE Communications Letters, vol. 10, no. 10, pp 701-703, 2006.

[14] S. Pilosof, et. al., "Understanding TCP fairnessover Wireless LAN," in Proc. IEEE Infocom 2003.

[15] R. Stanojevic, C. M. Kellett, and R. N. Shorten, "Adaptive Tuning of Drop-Tail Buffers for Reducing Queueing Delays," IEEE Communication Letters, vol. 10, no. 7, pp 570- 572, 2006.

[16] Thottan, and M. C. Weigle, "Impact of 802.11e EDCA on mixed TCP-based applications," in Proc. IEEE WICON 2006.

[17] Z. Zhao, S. Darbha, and A. L. N. Reddy, "A method for estimating the proportion of nonresponsive traffic at a router," IEEE/ACM Transactions on Networking, vol. 12, no. 4, pp. 708 718, 2004.

[18] DataTAG project, http://www.datatag.org.

[19] S. Floyd, "High Speed TCP for Large Congestion Windows," in RFC 3649, Experimental, December 2003.

[20] Katabi, M. Handley, and C. Rohrs, "Internet Congestion Control for Future High Bandwidth- Delay Product Environments," in Proceedings of ACM SIGCOMM'02, August 2002.

[21] Jin, D. X. Wei, and S. H. Low, "FAST TCP: Motivation, Architecture, Algorithms, Performance," in Proceedings of IEEE INFOCOM'04, March 2004.

[22] L. Xu, K. Harfoush, and I. Rhee, "Binary Increase Congestion Control for Fast, Long Distance Networks," in Proceedings of IEEE INFOCOM' 04, 2004.

[23] K. Tokuda, G. Hasegawa, and M. Murata, "Performance Analysis of High Speed TCP and its Improvement for High Throughput and Fairness against TCP Reno Connections," in Proceedings of at High Speed Network Workshop (HSN 2003), November 2003.

[24] T. Kelly, "Scalable TCP: Improving Performance in High speed Wide Area Networks," in ACM SIGCOMM Computer Communication Review, Volume 33, Issue 2, April 2003. 